\title{
Dificultades laborales de profesores en escuelas secundarias ${ }^{1}$
}

\author{
Fernando Agustín Flores
}

Universidad Nacional del Nordeste

(Argentina)

fernando_flores@yahoo.com.ar

\section{Resumen}

Se presentan resultados seleccionados de una investigación cuyo propósito fue caracterizar las dificultades que atraviesan profesores en sus primeros desempeños. El análisis se organiza según estos interrogantes: cuáles son las dificultades que enfrentan los profesores novatos en su labor docente en el aula, cómo las afrontan y qué modalidades de respuestas ofrecen.

El estudio fue de carácter exploratorio y de naturaleza cualitativa. La muestra quedó constituida por treinta profesores/as que se desempeñan en escuelas secundarias de Resistencia, Chaco (Argentina). Se emplearon dos cuestionarios semiestructurados.

Hallazgos significativos muestran que estos profesores afrontan las situaciones problemáticas, fundamentalmente, en el marco de múltiples procesos informales de aprendizaje, generados en los intercambios con colegas experimentados y en las propias experiencias exitosas ocurridas en el contexto de la escolaridad media.

$1 \quad$ En este artículo exponen los resultados seleccionados obtenidos de la investigación "Formación docente inicial y primeras experiencias laborales en escuelas de nivel medio", trabajo desarrollado como becario de investigación de la Secretaría General de Ciencia y Técnica de la Universidad Nacional del Nordeste, en estos últimos años.

Recepción: 2015-01-14 | Envío a pares: 2015-01-24 | Aceptación por pares: 2015-03-05 | Aprobación: 2015-03-15

DOI: 10.5294/edu.2015.18.3.3

Para citar este artículo / To reference this article / Para citar este artigo

Flores F., Agustín (2015). Dificultades laborales de profesores en escuelas secundarias. Educ. Educ., 18 (3), 411-431. DOI: 10.5294/ edu.2015.18.3.3 


\title{
Palabras clave
}

Formación de docentes en activo, escuelas secundarias, estrategias educativas, formación de profesores, Chaco-Argentina (Fuente: Tesauro de la Unesco).

\section{Labor Problems of High School Teachers}

\begin{abstract}
Selected results from a study designed to characterize the difficulties teachers face when starting their careers are presented in this article. The analysis is organized around the following questions: What difficulties do new, inexperienced teachers face in the classroom? How do they cope with those difficulties and what type of response do they offer? The study was exploratory and qualitative in nature. The sample was comprised of thirty male and female teachers who work at high schools in Resistencia, Chaco (Argentina). Two semi-structured questionnaires were used. The most significant findings show these teachers cope with problematic situations, fundamentally within the framework of multiple informal learning processes generated in exchanges with experienced colleagues and in their own successful experiences in the high school context.
\end{abstract}

\section{Key words}

In-service teacher training, high schools, teaching strategies, educating teachers, Chaco-Argentina (Source: Unesco Thesaurus). 


\section{Dificuldades de trabalho de professores em escolas secundárias}

Resumo

Apresentam-se resultados selecionados de uma pesquisa cujo propósito foi caracterizar as dificuldades enfrentadas pelos professores em seus primeiros trabalhos. A análise organiza-se de acordo com estas perguntas: quais são as dificuldades que os professores novatos enfrentam em seu trabalho docente na sala de aula, como as enfrentam e que modalidades de respostas oferecem. O estudo foi de caráter exploratório e de natureza qualitativa. A amostra foi constituida por 30 professores/ as que trabalham em escolas secundárias de Resistencia, Chaco (Argentina). Foram utilizados dois questionários semiestruturados. Descobertas significativas mostram que esses professores enfrentam situações problemáticas, fundamentalmente, no âmbito de múltiplos processos informais de aprendizagem, gerados nas trocas com colegas experientes e nas próprias experiências de sucesso ocorridas no contexto do ensino médio.

\section{Palavras-chave}

Formação de docentes ativos, escolas secundárias, estratégias educativas, formação de professores, Chaco-Argentina (Fonte: Tesauro da Unesco). 


\section{Introducción}

En este artículo se exponen los resultados obtenidos de la investigación "Formación docente inicial y primeras experiencias laborales en escuelas de nivel medio". Uno de sus objetivos generales es explorar y caracterizar las dificultades más frecuentes que atraviesan los profesores de Ciencias de la Educación y de Geografía en sus primeras experiencias laborales, y las modalidades de respuestas que emplean para afrontarlas. El análisis se ubica en las situaciones problemáticas que afrontan los profesores en los inicios de la docencia, referidas a dificultades que surgen en el trabajo cotidiano del docente en el aula, al tiempo que se examinan las modalidades de respuestas que construyen estos profesores cuando afrontan tales dificultades. Finalmente, el conjunto de estas modalidades de respuestas se integra para interpretarlas en términos de estrategias socioprofesionales de inserción laboral.

Diversos autores de este país y del exterior, al hablar de la formación docente, acuerdan en que el aprender a enseñar es un proceso de larga duración que incluye pautas de comportamientos incorporadas desde las primeras experiencias como alumnos en la trayectoria escolar previa, luego en la formación específica o de grado, y por último, en la socialización laboral en donde los graduados se desempeñan (Zoppi, 2001). Entendida en estos términos, Marcelo (1999) clasifica la formación del profesorado en cuatro fases: Preentrenamiento, involucra aquella influencia que el profesor ha vivido como alumno en experiencias escolares tempranas. Formación inicial, referida a la preparación formal en instituciones específicas. Iniciación, es la fase que abarca las primeras incursiones docentes en las escuelas. Finalmente, la fase de formación permanente, se da a lo largo de la vida profesional para el perfeccionamiento de la enseñanza, transcurridos los tres primeros años. Son distintos ámbitos de formación, a los que Davini concibe como: "ambientes de modelación de las prácticas y del pensamiento, de instrumentación de estrategias de acción técnico- profesionales y de desarrollo de las formas de interacción socioprofesionales" (1995, p. 79).

Asumiendo esta perspectiva evolutiva y dinámica del desarrollo profesional del profesor, en este estudio resultan de particular interés los primeros años de experiencia laboral docente, los mismos constituyen un periodo con unas características especiales y propias, profusamente analizadas por la literatura especializada (Zeichner, 1993; Huberman, 1989; Esteve, 1997; Alonso, 2001; Marcelo, 2007, entre otros autores). Desde esta perspectiva, se pretende remarcar que en el camino de convertirse en profesor, la iniciación en la docencia configura una etapa diferenciada y determinante para el desarrollo profesional subsiguiente. Es llamativo que, aunque la investigación sobre el profesorado novel es actualmente uno de los tópicos más investigados en el campo de la formación del mismo, nuestra región cuenta con escasas referencias de estudios que remitan a esta problemática.

Se considera etapa de profesor novel, siguiendo la conceptualización expuesta por Imbernon (1994), a la que transcurre durante los tres primeros años de ejercicio profesional, aunque hay autores que la extienden hasta los cinco. En algunos estudios, esta etapa de inducción o socialización a la profesión aparece dividida en una primera fase de umbral o antesala (seis meses) y una segunda de madurez y crecimiento que comprende el tiempo posterior (Vera, 1988). Por su parte, Cornejo Abarca (1999) concibe esta fase de la carrera profesional docente como una inducción, en la que distingue dos momentos claves: ambientación y progresión, enfatizando que se trata de un proceso en el hacerse profesional que, para los profesores recién titulados que ingresan al mundo laboral, ocurre en relación con una transición experimentada desde la situación de ser un sujeto en formación hasta la de convertirse gradualmente en un profesional autodirigido. Expresado en otros términos, es una etapa de socialización, en la que el nuevo profesor se integra como miembro activo y participante en el colectivo profesional. 
En la bibliografía se encuentran amplias referencias a las problemáticas del profesorado novel y de su proceso de socialización laboral. Es de interés aun a pesar del tiempo transcurrido, exponer los problemas comunes presentes en el profesorado novel identificados en la clásica obra de Veeman (1988), quien los sitúa en diferentes ámbitos: la disciplina en el aula, la motivación de los alumnos, su evaluación, la organización del trabajo en clase, la sobrecarga de trabajo, la falta de tiempo para preparar las clases (programación diaria), el dominio de los diferentes métodos de enseñanza y de la materia, la determinación del nivel de aprendizaje de los alumnos, los insuficientes e inadecuados materiales didácticos, las relaciones con los padres y con los otros profesores, el trabajo burocrático, las relaciones con los directivos, la falta de tiempo libre, la falta de apoyo y orientación, y, por último, el número de alumnos por aula. Por otra parte, Esteve, Franco y Vera (1995), llevaron a cabo una investigación aplicando un cuestionario a 325 profesores noveles. Estos autores encontraron que los principales problemas identificados por los profesores se referían a las condiciones de trabajo: carencia o defectos del material disponible, calidad de los locales, excesivo número de alumnos por aula, tareas de preparación del trabajo escolar y horario (1995). En la actualidad, distintos trabajos mencionan como dificultades prevalecientes que enfrentan los profesores principiantes: el manejo de grupo, el desarrollo de la enseñanza y las tareas administrativas (Serra, Krichesky y Merodo, 2009).

No obstante, más que centrar el examen en la naturaleza de los problemas docentes aludidos, es de interés destacar en este artículo las estrategias que los profesores desarrollan para afrontarlos. En tal caso, para esta ocasión lo crucial es el proceso de socialización profesional que experimentan los profesores noveles. Particularmente, a partir de estas primeras experiencias laborales en escuelas de nivel medio, los profesores debutantes empiezan a asumir y consolidar las pautas y características de una determinada cultura profesional. En tal sentido,
Contreras (1987), afirma que el profesorado inicia su tarea profesional con una tendencia a solucionar las situaciones problemáticas a través del aprendizaje vicario o aprendizaje fruto de la observación, que se basa en la imitación de los profesionales más cercanos a él. En efecto, en esta producción se analizan la naturaleza de estos aprendizajes adquiridos, las formas de adquisición y los efectos que ello puede producir en el proceso de desarrollo profesional de los profesores implicados. Para Imbernón (1994), si esa socialización se produce de forma aislada, implica percibir los problemas educativos de determinada manera, muy particular, y puede producir cambios en la conducta, las actitudes y la personalidad del profesor debutante. Asimismo, cuando tal experiencia es negativa, puede provocar en este un replanteamiento prematuro de su permanencia en el ejercicio docente. $\mathrm{O}$ bien, ante situaciones percibidas como amenazantes, los sujetos pueden emplear ciertos mecanismos de defensa para manejar los problemas que afrontan y así, en cierto modo, sobrevivir laboralmente a esta etapa.

Estos profesores, en sus primeros desempeños, comienzan a enfrentar dificultades $y$, a su vez, a elaborar y aplicar estrategias para intentar resolverlas. Estas estrategias se asumen a través de prácticas de ensayo y error, o bien se toman y reproducen aquellas que ya han sido probadas con éxito por sus colegas más experimentados. De modo tal, que se comienza a instalar en su actuar profesional cierta práctica empírica, desprovista de aquella capacidad reflexiva y de impulso renovador que acompaña a todo proceso de integración propiamente profesional, quedando reducida a ser una mera socialización adaptativa. Estos procesos que se orientan a la reproducción de tradiciones educativas han sido documentados reiteradamente, entre otros, por Rockwell: "en otro nivel, menos público, los profesores se comunican las anécdotas de experiencias pasadas y los incidentes que acaban de suceder con su grupo. Se intercambian recomendaciones prácticas y reflexiones propias sobre la labor docente" (1987, p. 17). 
Por su parte, Marcelo (2007) sostiene que la imitación acrítica de conductas observadas en otros profesores, el aislamiento de sus compañeros, la dificultad para transferir el conocimiento adquirido en su etapa de formación inicial y el desarrollo de una concepción técnica de la enseñanza son problemáticas de urgente tratamiento que afectan de modo negativo el proceso de socialización laboral de los profesores principiantes. Además, agrega, que no puede llevarse a cabo aprendizaje constructivo y reflexivo cuando otros elementos interfieren para disminuir la facultad de aprender: inseguridad, carencia de experiencia, falta de criterio al valorar las situaciones, etc. Dejados a su propia suerte, mal preparados desde su formación de grado y recibiendo escaso apoyo de parte de los otros miembros de la institución, se ven expuestos a una socialización poco favorable para una formación y desarrollo propiamente profesional.

Es entonces cuando se vuelve relevante conocer qué ocurre en el periodo de socialización de los profesores debutantes, con la cautela de que la socialización del profesor no es meramente un fenómeno de ajuste pasivo a las ideas y conductas normativas exigidas por la situación en la que participan, sino que el profesor, ubicado en la intersección de la biografía y la situación social, tiene algún margen para manipular y generar cambios en la situación, aunque al mismo tiempo está siendo presionado para ajustarse a ella.

De acuerdo con las afirmaciones clásicas de Lacey (1977), los profesores no se acomodan a la exigencia de su experiencia docente de un modo indiferente, por el contrario, desarrollan lo que él mismo ha denominado estrategias sociales, esto es, sistemas de ideas y acciones desarrolladas intencionalmente por un individuo en el contexto de una situación específica. Estas estrategias las selecciona el sujeto en función de la interpretación que hace de la situación y de la capacidad que tiene para llevarlas a cabo. Se pueden distinguir tres estrategias fundamentales: a. Conformidad estratégica, en la que el profesor se muestra conforme con la definición que hace la autoridad y con las restricciones que aquella impone, pero mantiene sus reservas privadas.

b. Ajuste interiorizado, es la estrategia que utilizan los profesores principiantes que asumen como propios los valores, las metas y las limitaciones de la institución. Desde esta perspectiva, no se produce ningún tipo de conflicto durante el proceso de socialización al existir sintonía entre el profesor, y los valores y las normas institucionales.

c. Redefinición estratégica de la situación, es la estrategia menos común y significa conseguir el cambio, impulsándolo directamente o capacitando a los que tienen el poder formal para cambiar la interpretación que estos tienen de lo que está sucediendo en una situación.

De esta forma, los profesores noveles, en función de la experiencia que les toque vivir y los problemas que enfrenten, desarrollan algún tipo de estrategias. Como bien lo expresa Contreras (1987), el modo de interpretar la interacción entre las intenciones individuales y las restricciones institucionales puede ayudarnos a comprender cómo el factor fundamental de socialización del profesor está no solo en la medida en que se ajustan a las exigencias institucionales, sino en cómo aprenden a imaginar sistemas de ideas y acciones que les permitan orientar su forma de actuar.

Por tanto, se puede afirmar, en concordancia con los estudios mencionados, que los profesores en sus primeros años de experiencia docente se encuentran con problemas prácticos del aula (organizar las actividades de aprendizaje, evitar problemas de disciplina, seleccionar y ordenar el contenido, etc.), y de la institución (presiones y exigencias ejercida por el director, otros profesores, los padres de los alumnos, etc.). La intervención del docente sobre estas dificultades genera procesos de actuación que 
pueden convertirse en una socialización adaptativa, de supervivencia profesional al medio, apareciendo situaciones de adaptación alienante, un ajuste estratégico interiorizado sin conflicto, aceptando las pautas de la institución, o bien, una adaptación estratégica con reservas o, en menor grado, una reacción o redefinición estratégica con conflicto al intentar cambiar las pautas colectivas instituidas.

\section{Aspectos metodológicos}

Abordaje metodológico cualitativo de carácter exploratorio, con el fin de interiorizar un proceso complejo en un contexto específico.

En el estudio participaron treinta profesores recién graduados y abocados al ejercicio de la docencia en el nivel medio: quince de Ciencias de la Educación y quince de Geografía, titulados en la Facultad de Humanidades de la Universidad Nacional del Nordeste (tabla 1).

Los criterios para la selección de la muestra fueron:

a. Que tuvieran como mínimo un año de ejercicio profesional y tres como máximo.

b. Oue los establecimientos escolares en donde se desempeñaran los profesores noveles estuvieran ubicado en el área de Gran Resistencia.

c. Qué fueran graduados de la Facultad de Humanidades de la UNNE.
A diferencia de los profesores de Geografía, los de Ciencias de la Educación tenían a cargo diferentes asignatura. Por ejemplo, impartían clases de Psicología, Filosofía, Formación Ética y Ciudadana, Sociología, y proyectos de definición institucional.

La estrategia de recolección de datos estuvo conformada por la integración de cuatro procedimientos. Tres destinados a captar información sobre las dificultades comunes que afectan el desempeño profesional de los profesores noveles y la manera como estos construyen modalidades de respuestas para afrontarlas, y uno con el propósito de recuperar las apreciaciones de los profesores pertenecientes a la institución formadora sobre las posibles relaciones de los problemas identificados y las modalidades de respuestas construidas con la formación inicial.

En este artículo se presentan y discuten resultados procedentes de la aplicación de los dos primeros instrumentos de recolección de información, a saber:

- Cuestionario semiestructurado. Contiene preguntas de respuesta abierta y de respuestas precategorizadas. Se incluyeron dimensiones problemáticas de la práctica docente referidas a su trabajo y a sus condiciones de realización: Enseñanza / Tiempo/ Personal / Relaciones/Entorno/Formación. La finalidad fue obtener información respecto al conjunto de dificultades más recurrentes que afectan a los

Tabla 1. Composición de la muestra

\begin{tabular}{|c|c|c|c|c|c|}
\hline $\begin{array}{c}\text { Profesores } \\
\text { principiantes }\end{array}$ & $\begin{array}{c}\text { Tiempo en la } \\
\text { doc. }\end{array}$ & Cant. de doc. & $\begin{array}{c}\text { Cant. de } \mathbf{h} \\
\text { didácticas }\end{array}$ & Situación de revista & $\begin{array}{c}\text { Cant. de } \\
\text { escuelas }\end{array}$ \\
\hline \multirow{2}{*}{$\begin{array}{c}\text { Profesores de } \\
\text { Geografía (15) }\end{array}$} & 1 año & 4 & $5-15 \mathrm{~h}$ & Suplente & $1-3$ \\
\cline { 2 - 7 } & 2 años & 4 & $15-25 \mathrm{~h}$ & Suplente/ interino & $1-3$ \\
\hline \multirow{2}{*}{$\begin{array}{c}\text { Profesores de } \\
\text { Ciencias de la } \\
\text { Educación (15) }\end{array}$} & 3años & 7 & $20-30 \mathrm{~h}$ & Suplente/ interino & $1-3$ \\
\cline { 2 - 7 } & 2 años & 3 & $2-10 \mathrm{~h}$ & Suplente & $1-2$ \\
\hline
\end{tabular}


profesores en los tres primeros años de experiencia en la docencia.

- Completar frases. Consiste en la presentación de frases de final incierto (enunciados incompletos), utilizadas para indagar sobre las principales modalidades de respuesta usadas por los profesores para hacer frente y resolver las dificultades presentes en su práctica escolar cotidiana.

Para el tratamiento de la información se ha privilegiado un análisis de doble naturaleza: de carácter cuantitativo y cualitativo, cuyo contenido fue integrado. Este análisis se focaliza en la caracterización de las dimensiones y los aspectos que se han mostrado como manifestaciones significativas en el abordaje de la problemática, destacando aquellas dificultades que afrontan los profesores principiantes de ambas carreras, ya sea en sus aspectos diferenciados así como en los similares, sus particulares modos de responder a ellas, recuperando sus voces acerca de cuáles son las estrategias más eficaces para una actuación exitosa.

\section{Resultados}

Del conjunto de las dificultades percibidas por los profesores en sus tres primeros años de ejercicio de docencia, se seleccionaron para esta publicación aquellos aspectos problemáticos que hacen alusión a la programación de la asignatura, la disciplina que se imparte, los recursos didácticos y la organización del tiempo y los espacios áulicos, las estrategias de enseñanza, la evaluación, la relaciones docente-alumno y entre colegas. Asimismo, se describen los modos de respuestas que se construyen para afrontarlos, luego se interpretan estos modos de enfrentar dificultades según el encuadre teórico de las estrategias de socialización laboral.

\section{Dificultades y modalidades de respuesta}

\section{¿Cuáles son las dificultades más comunes en la planificación de la enseñanza?}

Los profesores noveles señalan el grado de dificultad que les ocasiona un determinado conjunto de aspectos referidos a la planificación de la enseñanza, como se observa en la tabla 2. Los profesores de Geografía, así como los de Ciencias de la Educación, ubican aspectos relacionados con la planificación de la enseñanza en opciones intermedias de dificultad, según la escala expuesta en el cuestionario. Al tiempo que en el item de respuesta abierta, los de Geografía registraron dificultades relativas a los escasos recursos didácticos y la falta de información estadística actualizada. Por su parte, los de Ciencias de la Educación remarcaron la dificultad que surge al implementar lo diseñado, acrecentándose en actividades de realización extraclase. Ambos grupos de profesores coinciden en señalar dificultades referidas al trabajo aislado.

Tabla 2. Grados de dificultad de aspectos relacionados con la planificación de la enseñanza, según los señalamientos de ambos grupos de profesores noveles

\begin{tabular}{|c|c|c|c|c|c|c|c|c|}
\hline & \multicolumn{4}{|c|}{ Prof. de Geografia } & \multicolumn{4}{|c|}{ Prof. Ciencias de la Educación } \\
\hline & MD & AD & PD & ND & MD & AD & PD & ND \\
\hline a Selección y organización del contenido & - & 5 & 7 & 3 & - & 4 & 8 & 3 \\
\hline b. Formulación de objetivos & - & 3 & 9 & 3 & 1 & 3 & 7 & 4 \\
\hline c. Interdisciplinariedad & - & 6 & 4 & 5 & 2 & 5 & 7 & 1 \\
\hline d. Diseño de actividades de aprendizaje & - & 1 & 4 & 10 & 1 & 3 & 6 & 5 \\
\hline e. Otros & \multicolumn{4}{|c|}{$\begin{array}{c}\text { No contar con recursos didácticos } \\
\text { Trabajo aislado } \\
\text { Falta de información estadística }\end{array}$} & \multicolumn{4}{|c|}{$\begin{array}{c}\text { Resistencia de los alumnos ante } \\
\text { tareas innovadoras } \\
\text { Cumplir con los objetivos } \\
\text { Implementar las actividades de } \\
\text { aprendizaje Actividades en casa } \\
\text { Trabajo individualizado }\end{array}$} \\
\hline
\end{tabular}




\section{¿Cómo afrontan ambos grupos de docentes tales dificultades?}

Predominantemente, los profesores de Ciencias de la Educación proceden de dos maneras: algunos buscan apoyo institucional, acuden a la figura del asesor pedagógico, directivos y colegas. Destaca en estos profesores la buena relación, la comunicación fluida y el alto compromiso institucional. Otros, en cambio, con un modo de proceder individual, hurgan entre los apuntes y material bibliográfico que conservaron del profesorado, buscan algo que los pueda orientar y que les sirva con fines prácticos. Algunos de estos profesores consideran que las dificultades que afrontan tienen que ver con sus primeros pasos en la docencia y que con el transcurso del tiempo van a poder mejorar su accionar: "falta de tiempo y experiencia en la docencia”, "confío que el próximo año haré una propuesta más adecuada".

Los profesores de Geografía resuelven las dificultades originadas en la planificación recurriendo a otros profesores del área o a la asesora pedagógica, si la escuela posee una. Algunos profesores, cuando se les presenta dificultad, acuden a buscar información especializada sobre la temática —-Internet aparece como una fuente privilegiada y vía ágil y actualizada para obtener conocimientos, reconociendo en particular la utilidad de tener acceso a las publicaciones de especialistas en didáctica específica-.

Asimismo, ambos grupos de profesionales novatos buscan resolver aquellas dificultades relacionadas con la implementación de lo planificado mediante su propia iniciativa, ya sea buscando apoyo en sus colegas u otras figuras institucionales o bien a través de la relectura y el estudio de lo dado en el profesorado; en todos los casos, no se percibe que estos ámbitos escolares en donde se desempeñan propongan o posean un dispositivo colectivo que atienda estas problemáticas.

\section{¿Cómo hacer para organizar el contenido de la asignatura de un modo que al alumno no le resulte tan lejano a sus intereses?}

Se observa acuerdo en la mayor parte de estos profesores al señalar como aspectos problemáticos vinculados a la asignatura que se imparte: hacerla interesante para el alumno, realizar estudios de aplicación a la realidad y organizar los contenidos por enseñar según intereses y experiencias de los estudiantes. Diferencialmente, en los aspectos referidos al manejo sólido del contenido y a la presentación creativa de la asignatura, los profesores de Ciencias de la Educación reconocieron mayores dificultades de las que se perciben en los señalamientos de los de Geografía. En la pregunta de elaboración libre, los profesores de ambas especialidades, compartieron la dificultad generada por la apatía o el desinterés de los alumnos (tabla 3).

¿Cómo intentan resolver estas dificultades?: se abordó la problemática del desinterés de los alumnos por el contenido de la asignatura. Una respuesta común de ambos grupos de profesores, se refiere a que ellos apelan principalmente a generar variantes en la estrategia de enseñanza, empleando diversos recursos didácticos para apoyar sus clases: documentales, películas, empleo de las netbook, etc. Sostienen que estas acciones son favorables en el aprendizaje de los alumnos porque ellos retienen con mayor facilidad la información a través de la memoria visual. Un docente de Geografía señaló que le da buen resultado ir variando las actividades ya que esto predispone anímicamente de otra manera al alumno: "...los chicos se entusiasman cuando les traes propuestas nuevas... hay que tener en cuenta no solo el contenido, sino además el estado anímico del alumno, debemos tratar de que nuestras clases no sean aburridas" (Entrevista con Gastón, 08/05/2012).

Particularmente, a los de Geografía también les resulta muy productivo hacer visitas guiadas, construir un anecdotario, trabajar con recortes periodísticos, organizar ferias de ciencia, promover pequeños 
ISSN 0123-1294 | Educ.Educ. Vol. 18. No. 3 | Septiembre-Diciembre de 2015 | pp. 411-431.

Universidad de La Sabana | Facultad de Educación

Tabla 3. Grados de dificultad que ocasionan aspectos referidos a la asignatura que se imparte

\begin{tabular}{|c|c|c|c|c|c|c|c|c|}
\hline & \multicolumn{4}{|c|}{ Prof. de Geografía } & \multicolumn{4}{|c|}{ Prof. Ciencias de la Educación } \\
\hline & MD & AD & PD & ND & MD & $A D$ & PD & ND \\
\hline a. Dominar sólidamente los contenidos & - & - & - & 15 & - & 5 & 6 & 4 \\
\hline b. Presentar la enseñanza de forma creativa & - & 1 & 5 & 9 & 3 & 6 & 4 & 2 \\
\hline $\begin{array}{l}\text { c. Adaptar los contenidos a los intereses y las } \\
\text { expectativas de los diferentes grupos de alumnos }\end{array}$ & 4 & 4 & 7 & - & 2 & 5 & 5 & 3 \\
\hline $\begin{array}{l}\text { d. Organizar actividades y prácticas de aplicación a la } \\
\text { realidad }\end{array}$ & 7 & 2 & 6 & - & - & 5 & 8 & 2 \\
\hline e. Hacer atractiva la asignatura & - & 6 & 6 & 3 & 3 & 7 & 4 & 1 \\
\hline f. Otros & \multicolumn{4}{|c|}{$\begin{array}{l}\text { La velocidad de la información } \\
\text { transmitida por los medios de } \\
\quad \text { comunicación } \\
\text { Apatía generalizada de los octavos } \\
\text { años hacia la asignatura }\end{array}$} & \multicolumn{4}{|c|}{$\begin{array}{l}\text { Falta de continuidad en el dictado de } \\
\text { la materia por paros, jornadas, etc. } \\
\text { Apatía de algunos alumnos. } \\
\text { Inasistencia o deserción de los } \\
\text { alumnos }\end{array}$} \\
\hline
\end{tabular}

trabajos de investigación. Otro profesor agregó que para estimular el interés de los estudiantes recurre frecuentemente a juegos competitivos.

En cambio, un grupo de profesores, predominantemente de Ciencias de la Educación, pone el foco en los contenidos por desarrollar. Sostienen que despertar el interés de los alumnos requiere organizar el contenido de modo tal que esté directamente relacionado con la realidad del alumnado, o con temas que los movilicen. A propósito de esto comentó:

...en un noveno enseño técnicas de estudio... les pedi directamente a los alumnos que me dijeran lo que realmente les interesaría... los incité a que seleccionen un tema que de algún modo les interesase. Asi fue que un grupo seleccionó sobre el maltrato infantil, otro sobre alcoholismo, y otro acerca de la drogadicción, etc... se interesaron bastante y anduvieron muy bien en la confección de las monografias (Entrevista con Walter, 10/05/2012).

Una profesora de Ciencias de la Educación, que dicta clases de Filosofía, reveló que obtuvo buenos resultados trabajando del siguiente modo: ...realmente los chicos se prestan y entusiasman mucho [...] cuando el tema se presta les pido que relaten algunas experiencias vividas o sino yo misma les consigo relatos para que reflexionen sobre ellos, por ejemplo, cuando se abordó la cuestión ética: los criterios y las normas del ac cionar humano, les había presentado algunos casos de la vida real para que traten de hacer una evaluación ética de los mismos, es decir tenían que intentar aplicar criterios éticos desarrollados durante las clases, o bien propongan valoraciones éticas de estas situaciones siguiendo las conceptualizaciones elaboradas en las clases teóricas (Entrevista con Mabel, 30/04/2012).

¿Cómo organizar los recursos disponibles en la escuela, y manejar el espacio y el tiempo áulicos para promover aprendizajes?

Coincidentemente, los profesores noveles señalaron que poseen entre mucha y alguna dificultad al utilizar recursos audiovisuales y otras tecnologías en el aula, y contar con la infraestructura y los materiales para la enseñanza de la materia. Además, los profesores en Ciencias de la Educación 
señalaron dificultades relacionadas con la organización y distribución del tiempo y el espacio para el trabajo en clase. En la pregunta de respuesta abierta, ambos grupos de profesores reconocen marcadas limitaciones localizadas en la falta de recursos de todo tipo para apoyar la enseñanza (tabla 4).

Entonces, ¿cómo afrontan las dificultades relacionadas con los recursos didácticos y la organización del tiempo y los espacios áulicos?

Entre las respuestas existe acuerdo en plantear que estas dificultades se originan por carencias y ausencia de recursos institucionales, así como por falta de apoyo gubernamental; también, en cuanto al uso de las nuevas tecnologías de la información y la comunicación existen deficiencias en la formación docente. Sin embargo, algunos profesores sostienen que la situación descrita no es un impedimento; por ejemplo, una profesora comentó que la escuela en donde trabaja no posee sala de informática, y si bien está dentro del programa Conectar Igualdad — por lo que comenzó a recibir las primeras netbook-, aún no posee conexión a Internet y el piso tecnológico no funciona. Ante esta dificultad, solicitó autorización en un Ciberg del barrio y fue a trabajar con los alumnos a ese lugar. Otro docente manifestó que para desarrollar con algunas variantes las clases, consigue DVD y TV prestado o facilitado por los mismos alumnos.

Nuevamente, aquí se advierte que la resolución de las dificultades depende de la iniciativa de los docentes, de sus ganas de asumir otras responsabilidades, de su tiempo, y de la colaboración de los alumnos que poseen estos recursos y que tienen la intención de facilitarlos a la institución.

\section{¿Cómo integrar y emplear las TIC en las propuestas de enseñanza?}

Coincidentemente, los profesores señalaron distintos grados de dificultad para conseguir que los estudiantes logren utilizar las nuevas tecnologías, así como despertar el interés de los estudiantes por aprender y, a su vez, llevar a cabo estrategias innovadoras en el aula.

Llamativamente, el grupo de profesores en Ciencias de la Educación, contrario a los de Geografía, señaló mayores dificultades en el conjunto de aspectos referidos a las estrategias de enseñanza, a pesar de poseer formación especializada.

Para la pregunta de respuesta abierta, se expusieron dificultades relativas a las carencias económicas de los alumnos y a las continuas inasistencias de los mismos, ambos aspectos restrictivos a la hora de desarrollar estrategias de enseñanzas (tabla 5).

Ahora bien, ¿cómo intentan resolver las dificultades relativas a esta cuestión?

La mayor parte de estos profesores, contrariamente a lo esperado, manifiestan que la estrategia de enseñanza que brinda mejores resultados consiste en una clara exposición del tema que se va a desarrollar, o sea una clase magistral tradicional, aunque con algunos atenuantes, tales como: crear un espacio para que los alumnos puedan ir expresando las dudas que les van surgiendo, que el docente sea lo suficientemente flexible para abrirse al diálogo y al debate de ideas, que sea capaz de solicitar trabajos grupales de distintos tipos ya sean escritos $u$ orales -los docentes entienden que el trabajo en equipo potencia los aprendizajes-. Al respecto, una profesora de Ciencias de la Educación expresó:

...conformar grupos y brindar material de lectura, pedir que realicen un cuadro sinóptico, y después explicar. Muchas veces sirve pasar grupo por grupo a monitorear el trabajo, identificar a quiénes les cuesta más y realizar explicaciones más individualizadas (Entrevista con Silvia, 10/06/2012).

Un docente de Geografia sostiene que no puede hacer trabajar a sus alumnos en grupo: "son muy inquietos, entonces no me queda otra alternativa que dictarles el tema, es la única forma en que puedo hacerles trabajar. Se resisten a las propuestas innovadoras" (Entrevista con Hugo, 15/05/2012). 
Tabla 4. Grados de dificultad que causan aspectos vinculados con los recursos didácticos y la organización de la clase

\begin{tabular}{|c|c|c|c|c|c|c|c|c|}
\hline & \multicolumn{4}{|c|}{ Prof. de Geografia } & \multicolumn{4}{|c|}{ Prof. Ciencias de la Educación } \\
\hline & MD & $A D$ & PD & ND & MD & AD & PD & ND \\
\hline a. Organizar y distribuir el tiempo del aula & - & 2 & 7 & 6 & 2 & 2 & 9 & 2 \\
\hline b. Organizar el espacio del aula & - & 2 & 4 & 9 & 2 & 1 & 6 & 6 \\
\hline $\begin{array}{l}\text { c. Utilizar recursos audiovisuales y otras tecnologías } \\
\text { en el aula }\end{array}$ & 7 & 5 & 2 & 1 & 5 & 6 & 3 & 1 \\
\hline $\begin{array}{l}\text { d. Contar con la infraestructura y los materiales para } \\
\text { la enseñanza de la materia }\end{array}$ & 3 & 7 & 4 & 1 & 4 & 6 & 5 & - \\
\hline e. Otros & \multicolumn{4}{|c|}{$\begin{array}{c}\text { No contamos con los recursos } \\
\text { audiovisuales ni la infraestructura } \\
\text { para enseñar la materia } \\
\text { No se cuenta con el espacio físico } \\
\text { adecuado } \\
\text { La falta de apoyo de los gobernantes } \\
\text { limita el accionar de las escuelas }\end{array}$} & \multicolumn{4}{|c|}{$\begin{array}{c}\text { No contamos con recursos } \\
\text { audiovisuales Escasa preparación en } \\
\text { el uso de ciertas tecnologías } \\
\text { La institución en la que me } \\
\text { desempeño carece de recursos } \\
\text { tecnológicos }\end{array}$} \\
\hline
\end{tabular}

Tabla 5. Grados de dificultad de aspectos referidos a las estrategias de enseñanza

\begin{tabular}{|c|c|c|c|c|c|c|c|c|}
\hline & \multicolumn{4}{|c|}{ Prof. de Geografía } & \multicolumn{4}{|c|}{ Prof. Ciencias de la Salud } \\
\hline & MD & AD & PD & ND & MD & AD & PD & ND \\
\hline a. Explicar los temas con claridad & - & - & 3 & 12 & - & 4 & 8 & 3 \\
\hline $\begin{array}{l}\text { b. Conseguir normas de funcionamiento de la clase } \\
\text { aceptadas o pactadas con el alumno }\end{array}$ & - & - & 4 & 11 & 2 & 5 & 5 & 3 \\
\hline $\begin{array}{l}\text { c. Organizar las actividades que el alumno tiene que } \\
\text { realizar }\end{array}$ & - & - & 3 & 12 & - & 2 & 6 & 7 \\
\hline $\begin{array}{l}\text { d. Preparar actividades prácticas y de aplicación a la } \\
\text { realidad }\end{array}$ & - & 4 & 4 & 7 & 3 & 3 & 6 & 3 \\
\hline e. Llevar a cabo estrategias innovadoras en el aula & - & 7 & 3 & 5 & 3 & 4 & 5 & 3 \\
\hline f. Elaborar material escrito y de apoyo para las clases & - & - & 2 & 13 & 4 & - & 9 & 2 \\
\hline $\begin{array}{l}\text { 9. Conseguir que los estudiantes logren utilizar las } \\
\text { nuevas tecnologías }\end{array}$ & 3 & 3 & 8 & 1 & 6 & 8 & - & 1 \\
\hline $\begin{array}{l}\text { h. Adaptarse a la diversidad existente entre los } \\
\text { estudiantes }\end{array}$ & - & 3 & 5 & 7 & 1 & 3 & 9 & 2 \\
\hline i. Crear interés y motivar a los estudiantes & - & 7 & 5 & 3 & 4 & 6 & 2 & 3 \\
\hline j. Otro & \multicolumn{4}{|c|}{ El nivel económico de los alumnos } & \multicolumn{4}{|c|}{$\begin{array}{l}\text { Las inasistencias de los alumnos } \\
\text { dificultan el cumplimiento de los } \\
\text { objetivos de diferentes etapas en e } \\
\text { desarrollo de los temas }\end{array}$} \\
\hline
\end{tabular}

Otro profesor de Geografía manifestó: "las clases magistrales son necesarias pero no siempre, trato de ir alternando; me gustan mucho los resultados que se obtienen cuando se organizan actividades de investigación con los chicos, se entusiasman mucho y más cuando se les reconoce su esfuerzo y se les brinda apoyo" (Entrevista con Hector, 05/07/2012).

Otro, esta vez de Ciencias de la Educación, hace referencia a que utiliza distintas estrategias, trata de no hacer siempre lo mismo, dice que es funda- 
mental conocer y dar respuestas a las necesidades e inquietudes de los alumnos.

Sacarlos del aula y proponer trabajos al aire libre suele generar buenos resultados, sostiene un profesor de Geografía: "ir al patio leer y sobre todo debatir es algo que les gusta mucho a los alumnos".

\section{¿Qué tipos de dificultades referidas a la evaluación se señalan con mayor frecuencia?}

Del conjunto de aspectos referidos a la evaluación (ver tabla 6), más de dos tercios de los profesores de Geografía coincidieron en señalar que disponer de conocimientos de criterios, técnicas y estrategias para evaluar la propia docencia les produce cierto grado de dificultad. Mientras que las demás alternativas fueron escasamente señaladas con algún grado de dificultad.

Si se observa la columna derecha de la tabla 6 , ocurre lo contrario, existe una distribución distinta de las menciones. Todas las opciones fueron señaladas alternando grados de dificultad por la mayoría de profesores en Ciencias de la Educación.
En la opción de respuesta abierta, los de Ciencias de la Educación agregaron las dificultades para autoevaluar su trabajo. En cambio, para los de Geografía es la falta de tiempo en la corrección.

Pero, ¿cómo resuelven los profesores estas dificultades?

Algunos profesores de Ciencias de la Educación tratan de que las situaciones de evaluación no sean vividas por los alumnos de modo traumático, ante todo buscan que exista un buen clima, que sea de confianza, que los alumnos opinen acerca de lo que están aprendiendo y pregunten si les quedan dudas. A su vez, para calificar el rendimiento de los alumnos se apoyan en los exámenes, aunque consideran fundamental la participación en clase; sobre todo valoran la construcción de las respuestas a las preguntas formuladas en clase, y se valora la asistencia continua. Los docentes que manifestaron no contar con suficiente tiempo para la corrección de exámenes se inclinan a que estos sean orales. Para estos profesores principiantes, existe en las instancias evaluativas una alta valoración hacia los aspectos

Tabla 6. Grados de dificultad en aspectos relativos a la evaluación

\begin{tabular}{|c|c|c|c|c|c|c|c|c|}
\hline & \multicolumn{4}{|c|}{ Prof. de Geografía } & \multicolumn{4}{|c|}{ Prof. Ciencias de la Educación } \\
\hline & MD & $A D$ & PD & ND & MD & $A D$ & PD & ND \\
\hline $\begin{array}{l}\text { a. Organizar el proceso y las actividades de evaluación } \\
\text { de la asignatura }\end{array}$ & - & - & 6 & 9 & - & - & 8 & 7 \\
\hline b. Identificar la preparación previa de los estudiantes & - & 2 & 5 & 8 & 2 & - & 8 & 5 \\
\hline c. Llevar a cabo una evaluación continuada & - & 1 & 6 & 8 & - & - & 8 & 7 \\
\hline $\begin{array}{l}\text { d. Seleccionar el tipo de prueba apropiada en cada } \\
\text { situación }\end{array}$ & - & - & 5 & 10 & - & 2 & 7 & 6 \\
\hline e. Utilizar estrategias alternativas de evaluación & - & 2 & 5 & 8 & 2 & 2 & 6 & 5 \\
\hline f. Corregir los exámenes de los estudiantes & - & 3 & 3 & 9 & - & 5 & 6 & 4 \\
\hline g. Evaluar los trabajos de campo & - & 2 & 5 & 8 & 1 & 1 & 9 & 4 \\
\hline $\begin{array}{l}\text { h. Utilizar la evaluación de los estudiantes para } \\
\text { mejorar el proceso de enseñanza-aprendizaje }\end{array}$ & - & - & 4 & 11 & 1 & 1 & 7 & 6 \\
\hline $\begin{array}{l}\text { i. Disponer de conocimientos, de criterios, técnicas y } \\
\text { estrategias para evaluar la propia docencia }\end{array}$ & - & 5 & 7 & 3 & 1 & 1 & 8 & 5 \\
\hline j. Confeccionar pruebas de evaluación & - & - & 6 & 9 & - & 3 & 6 & 6 \\
\hline k. Otros & \multicolumn{4}{|c|}{$\begin{array}{l}\text { Falta de tiempo para corregir } \\
\text { evaluaciones }\end{array}$} & \multicolumn{4}{|c|}{ Dificultades para la autoevaluación } \\
\hline
\end{tabular}


ISSN 0123-1294 | Educ.Educ. Vol. 18. No. 3 | Septiembre-Diciembre de 2015 | pp. 411-431.

Universidad de La Sabana | Facultad de Educación

que refieren a la predisposición de los alumnos, a su esfuerzo, aclaran que la nota producto del examen trimestral es solo una pequeña parte de la nota final. Sorprendentemente, solo uno manifestó que un aspecto relevante en la instancia evaluativa es la capacidad de relacionar los temas con la realidad y con temas dados previamente; además, remarcó como criterio importante la correcta utilización de términos técnicos.

Desde la mirada de los profesores de Geografía, los alumnos están en condiciones de aprobar la materia cuando evidencian dedicación y esfuerzo en el estudio, cuando intentan superarse; cuando muestran desenvolvimiento en el manejo de los contenidos, prolijidad en los trabajos solicitados, carpeta completa, buena redacción y ortografía, respeto por los compañeros y por el profesor; además, presentación en tiempo y forma de las actividades escritas que se solicitan. Sostienen que tratan de aplicar distintos tipos de exámenes aunque a veces se ven imposibilitados de hacerlo por el calendario.
Finalmente, solo uno de los profesores valora de forma positiva la capacidad de los alumnos de relacionar los conocimientos de modo significativo.

\section{¿Cómo mantener la disciplina en el aula y el interés en clase, claves en la relación entre docente y alumnos?}

Ambos grupos de profesores muestran amplias coincidencias y solo ciertas discrepancias en las indicaciones de aspectos asociados a grados de dificultad relativos a la relación docente-estudiantes. Como se observa en la tabla 7, coinciden en colocar como aspecto problemático: despertar y mantener el interés de los alumnos hacia la asignatura; asimismo, señalan que no tienen dificultad en establecer relaciones interpersonales con los estudiantes sin perder objetivos docentes. En los restantes aspectos se advierten señalamientos orientados a distintos grados dificultades. Estos grados se acentúan levemente en las menciones hechas por los profesores de Ciencias de la Educación.

\section{Tabla 7. Grado de dificultad de aspectos referidos al vínculo pedagógico que se establece entre docente-alumnos}

\begin{tabular}{|c|c|c|c|c|c|c|c|c|}
\hline & \multicolumn{4}{|c|}{ Prof. de Geografia } & \multicolumn{4}{|c|}{ Prof. Ciencias de la Educación } \\
\hline & MD & $A D$ & PD & ND & MD & $A D$ & PD & ND \\
\hline $\begin{array}{l}\text { a. Establecer relaciones interpersonales con los } \\
\text { estudiantes sin perder objetivos docentes }\end{array}$ & - & - & 3 & 12 & - & 3 & 2 & 10 \\
\hline b. Potenciar la participación del alumnado en la clase & - & 4 & 4 & 7 & 1 & 4 & 8 & 2 \\
\hline c. Tratar a los estudiantes de manera personalizada & - & 3 & 5 & 7 & 1 & - & 8 & 6 \\
\hline $\begin{array}{l}\text { d. Atender de forma individualizada las demandas y } \\
\text { necesidades de los estudiantes }\end{array}$ & - & 4 & 5 & 6 & 2 & 2 & 7 & 4 \\
\hline e. Contener a los alumnos y satisfacer sus intereses & - & 4 & 6 & 5 & 4 & 4 & 6 & 1 \\
\hline $\begin{array}{l}\text { f. Despertar y mantener interés de los alumnos hacia } \\
\text { la asignatura }\end{array}$ & - & 8 & 6 & 1 & 2 & 9 & 3 & 1 \\
\hline $\begin{array}{l}\text { 9. Controlar la conducta de los alumnos y la } \\
\text { asistencia regular a clases }\end{array}$ & - & 3 & 6 & 6 & 1 & 7 & 4 & 3 \\
\hline $\begin{array}{l}\text { h. Determinar el nivel de aprendizaje de los alumnos, } \\
\text { y establecer estrategias para atender diferencias en } \\
\text { los ritmos de aprendizaje }\end{array}$ & - & 1 & 7 & 7 & - & 5 & 9 & 1 \\
\hline i. Otros & \multicolumn{4}{|c|}{$\begin{array}{l}\text { Problemas familiares, personales y } \\
\text { económicos }\end{array}$} & \multicolumn{4}{|c|}{$\begin{array}{l}\text { Despertar interés y la asistencia } \\
\text { regular a clase } \\
\text { Falta de interés de las familias } \\
\text { Falta de apoyo institucional }\end{array}$} \\
\hline
\end{tabular}


En el ítem de respuesta abierta, los de Geografía resaltaron aspectos problemáticos vinculados con dificultades de tipo familiar, económicas y personales. Por su parte, los de Ciencias de la Educación hicieron lo propio remarcando cuestiones deficitarias de índole familiar y escolar.

Cuando se trató el modo de resolver estas dificultades, la mayor parte de los docentes de ambos profesorados mostraron preocupación por problemas de indisciplina que suelen ocurrir en clase; se habló de la importancia de establecer una buena comunicación con el grupo de alumnos, de buscar apoyo en los auxiliares docentes y de tener periódicos encuentros con los padres de los "chicos más problemáticos".

Una profesora comentó que ella tiene una estrategia para controlar a los alumnos, que consiste en colocar negativos, una especie de nota conceptual; cuando los estudiantes acumulan tres negativos se les consigna un punto menos en conducta que repercutirá en la calificación. Sostiene que esto le da buenos resultados y señaló que esta estrategia es una práctica bastante usual en la institución en donde trabaja. Otra profesora de Geografía mencionó que ante un un caso grave de indisciplina, lo que hizo fue derivar a un centro especializado en adolescencia; también se acude a sanciones establecida institucionalmente. Como caso curioso, un profesor contó lo que hizo un año para establecer el orden en la clase: identificó al líder del grupo y pautó con él algunos acuerdos informales para que le ayudara a llevar adelante la clase en cuanto a establecer el orden; era un grupo particularmente inquieto; la recompensa consistía en la compra de gaseosa o comida en el recreo a cambio de su colaboración.

\section{¿Cómo transformar las condiciones institucionales que enmarcan las relaciones entre profesores?}

Con la siguiente pregunta se indagó sobre las dificultades relativas a la relación laboral entre profesores: "¿Logra reconocer en la relación con sus colegas alguna dificultad que considere afecta su desempeño profesional?".

Entre las respuestas brindadas por los profesores de Geografía, las problemáticas referidas tenían que ver con la "falta" o "ausencia" de interés para participar en reuniones de departamento, de compromiso institucional, de responsabilidad para llevar adelante obligaciones escolares, de predisposición y de tiempo para concretar trabajos institucionales, etc. Por su parte, los profesores de Ciencias de la Educación plantearon como dificultades: la falta de comunicación entre los profesores, trabajo aislado (no se trabaja en equipo), falta de pertenencia del docente a la institución, falta de compromiso y responsabilidad para llevar adelante tareas institucionales básicas, falta de acuerdos institucionales, dificultades para instituir prácticas innovadoras y para coordinar trabajos con profesores de otras áreas, desinterés de los profesores para abordar la problemática de los alumnos y acordar acciones para intentar resolverlas.

Inversamente, la mitad de los profesores de Geografía señaló que no tiene dificultad en la relación con sus colegas. Incluso algunos mencionaron que la relación es muy buena, y observan que siempre están predispuestos a colaborar y a ayudar cuando se los necesita. Una profesora sostiene que siempre se apoya en los docentes con más años de antigüedad en el establecimiento ya que considera que son muy importantes por el aporte y los consejos que brindan. En ocasiones, manifiesta la docente, "cuando tengo algún inconveniente con los chicos, ellos (colegas con más tiempo en la institución) me dan útiles consejos para resolverlos".

Otros profesores reconocieron tener cierta dificultad en su institución con algunos profesores, pero como las dificultades fueron de índole personal, no dieron mayores detalles.

Solo tres profesores noveles de Ciencias de la Educación mencionaron que no tienen dificultad con sus colegas. Una profesora señaló que al ser 
nueva en la profesión, suelen aconsejarla, guiarla y ayudarla frecuentemente. Otros indicaron como dificultades la falta de comunicación de los profesores y el trabajo aislado de los colegas; en relación con esto un docente manifestó:

...la organización de nuestras instituciones y del sistema educativo en general no favorecen el trabajo en equipo, ni en red. Ya que el docente de nivel medio en general transita por varias instituciones reduciendo su tiempo de trabajo de integración e intercambio [...] A las instituciones les falta un trabajo de diagnóstico, seguimiento y evaluación permanente que permita trabajar estas problemáticas (Entrevista con Alejandro, 02/07/2012).

Otra dificultad grave que perciben estos profesores es la falta de pertenencia del docente hacia el establecimiento educativo en donde ejerce su labor; explican que esto produce falta de compromiso y responsabilidad, falta de comunicación, y en ocasiones se agrava llegando a la absoluta desorganización para llevar adelante tareas institucionales básicas. También, señalaron que el individualismo imperante en el trabajo docente, y el escaso sentimiento de pertenencia, son el resultado de la poca cantidad de horas de clases que particularmente los de Ciencias de la Educación disponen en las escuelas.

Otro docente resaltó que no se logran poner de acuerdo con sus colegas o aunar criterios de trabajo porque no aceptan las ideas nuevas, tienen concepciones fuertemente arraigadas que refieren a la enseñanza y al a prendizaje; primordialmente sostienen que no llegan a ningún acuerdo específicamente cuando hay que definir los objetivos generales y las cuestiones centrales del proceso evaluativo. En este sentido, una docente reconoció que la condición de recién iniciada en la profesión afecta su desempeño de modo negativo.

Otro profesor señaló que se le presentan dificultades cuando tiene que realizar actividades con profesores de otras áreas, percibe falta de comuni- cación ya sea por los horarios que dispone cada uno o también por el escaso interés que evidencian algunos para ocuparse de las problemática del alumnado; a decir de un docente: "existe cierto egoísmo en los profesores que dificulta la coordinación para el trabajo en equipo".

A continuación, la figura 1 sintetiza, en datos porcentuales, las distintas dimensiones problemáticas abordadas en el cuestionario con preguntas de respuestas precategorizadas.

\section{Las estrategias de inserción laboral}

En el apartado precedente se caracterizaron las dificultades prevalecientes en la inserción laboral de profesores principiantes: se describieron las problemáticas comunes que atraviesan profesores de Geografía y de Ciencias de la Educación, y las acciones llevadas a cabo para intentar resolverlas. En este apartado, se interpretan las diferentes respuestas de los docentes noveles, las cuales van conformando en ellos un conocimiento construido en la práctica que posibilita el desarrollo de estrategias para sobrevivir, adaptarse y desarrollarse en el ejercicio profesional (Serra et al., 2009).

Los aspectos problemáticos más salientes en el inicio de la actividad laboral de los docentes, detectados en este estudio, muestran más consistencias que contradicciones con resultados obtenidos en investigaciones que abordan esta misma cuestión.

Antes de avanzar sobre el par dificultad-respuesta en la perspectiva de los profesores noveles, se analiza brevemente qué se quiere decir cuando se habla de situaciones problemáticas vinculadas con el periodo que atraviesa este grupo profesional. Para tal fin, se recuperan los aportes de Evans, Llorente, Martínez, Otsubo, Rancaño, Sena y Strack, quienes entienden el concepto de problemática en el sentido de un quiebre que permite un aprendizaje; un conflicto sobre el que se debe hacer algo, abarca tanto la situación que conflictúa como también la respuesta dada en ese momento a dicha situación. Estos auto- 


\section{Figura 1. Porcentajes comparativos de dificultades referidas a distintas dimensiones de la actividad docente según profesores noveles de Geografía y de Ciencias de la Educación}

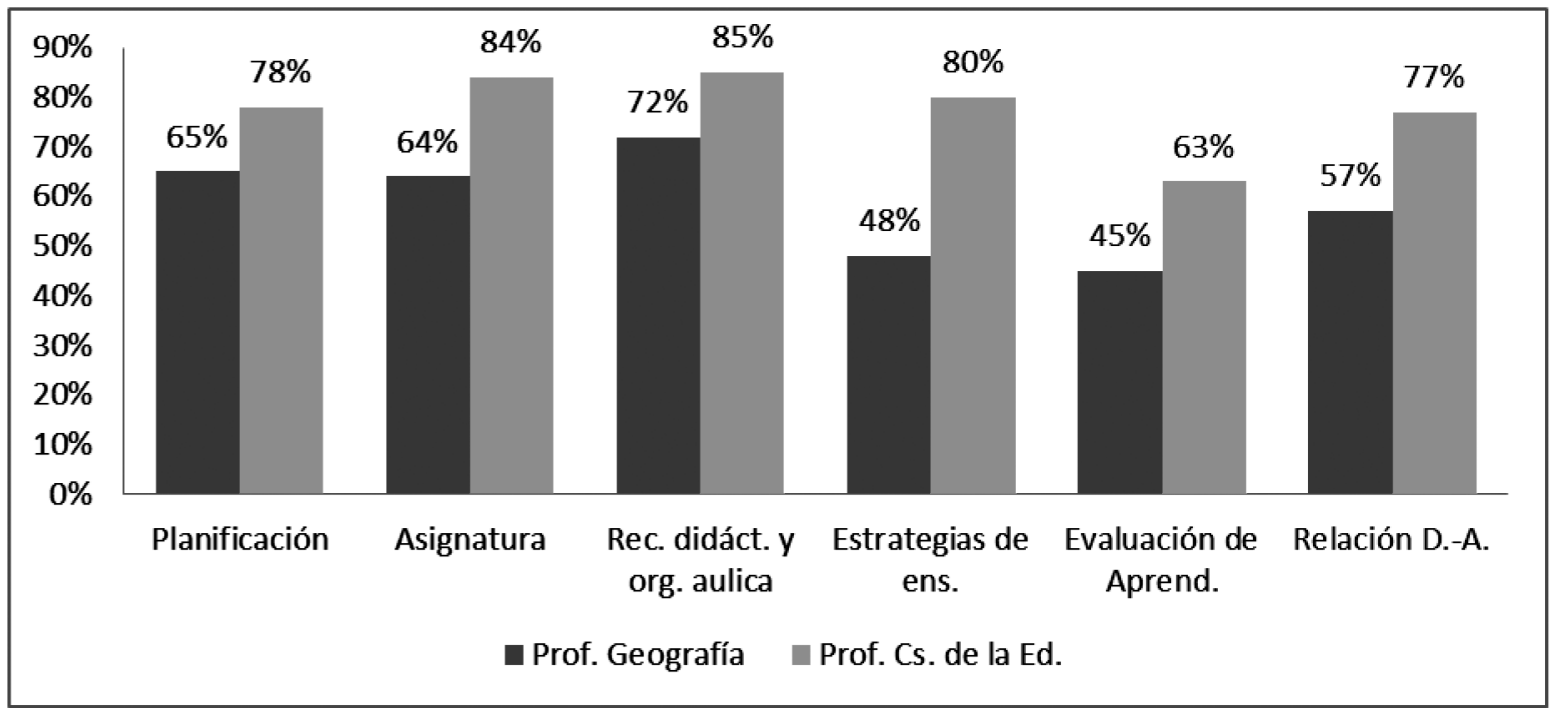

res sostienen que las problemáticas contienen una tensión implícita entre la práctica inicial laboral y la formación de la cual egresaron (Evans et al., 2010). En suma, sobre este escenario de tensiones que reporta esta investigación, el análisis caracteriza los procesos adaptativos que vivencian los profesores en el ejercicio inaugural de su profesión en el marco de instituciones escolares de nivel secundario.

Ahora bien, del conjunto de los aspectos problemáticos presentados, se advierte que estos profesores principiantes los afrontan prevalecientemente desde su iniciativa individual y no desde el encuadre de estrategias formativas planificadas con sustento institucional. Lo hacen de un modo informal y asistemático, sea a partir del apoyo en otros actores institucionales: buscando recomendaciones, sugerencias, recetas, etc.; generalmente, son profesores que mantienen una buena relación, fluida comunicación y colaboran permanentemente en tareas institucionales. Las figuras con quienes intercambian inquietudes son el asesor pedagógico, los directivos u otros profesores que generalmente llevan un tiempo más prolongado en la institución o en la docencia. Se revela entonces que el profesor elabora las estrategias mediante la interacción con otros actores institucionales. También, aparecen otros profesores con un proceder que se basa en la búsqueda en solitario de algo que les puede servir, ser útil en su práctica. En este caso, lo que hacen es recurrir a apuntes y a archivos de material bibliográfico de su formación de grado; otra fuente consultada es Internet, o incluso rastrean en su memoria biográfica experiencias escolares que se remontan a su rol de alumno. Si bien estos profesores, como se acaba de exponer, construyen respuestas de modo distintivo, ambos mantienen en común que operan desde la iniciativa individual. En este sentido, numerosos autores acuerdan en la necesidad de generar mecanismos de inserción formalizados con el propósito de convertir los primeros años de ejercicio docente en una fructífera experiencia formativa (González Brito et al., 2005; Marcelo, 1998 y 2007).

Een coincidencia con hallazgos presentados en el trabajo de Serra, Krichesky y Merodo (2009), estos profesores poseen la fuerte creencia de que con el transcurrir del tiempo y la acumulación de experiencia me- 
jorarán sustancialmente su desempeño profesional. De igual modo, los autores mencionados revelaron que en la percepción de los docentes novatos aparece la convicción de que la experiencia posibilita adquirir herramientas y, a la vez, confianza. Ambas cuestiones consideran los participantes del estudio indicado, contribuyen a generar intervenciones de enseñanza más pertinentes

Otra cuestión por destacar es el alto grado de dificultad que les ocasiona a los profesores convertir la presentación de los contenidos disciplinares en asequibles e interesantes para los alumnos. En relación con este aspecto problemático, por cierto resultado muy recurrente en diversos estudios, Bleichmar (1997) introdujo el concepto de malestar sobrante, de importante potencial interpretativo, que se puede emplear consistentemente para entender la apatía de los adolescentes, la falta de metas o de constancia para perseguirlas, en su visión desesperanzada del futuro o en el desinterés que muestran en las aulas. Esta noción de corte psicoanalítico hace referencia o está dada por una serie de factores característicos de cada época. Básicamente, los profesores noveles consultados responden de dos modos a esta dificultad, algunos ponen el foco de atención en la organización del contenido disciplinar para revertir este desinterés, mientras que otros ponen el énfasis en los materiales y recursos didácticos para apoyar y llevar adelante el desarrollo de las clases.

Respecto a las dificultades relativas a la carencia e insuficiencia de recursos y materiales en la institución para la enseñanza, generalmente los docentes consiguen estos recursos por su cuenta, ya sea porque disponen de los mismos o los consiguen por la colaboración de algún estudiante, por ejemplo, prestar un televisor o un DVD, en el caso que esté programado pasar una película. Es decir, el docente debe arreglárselas como pueda. Al respecto, uno de los cuatro modelos de inserción profesional formulados por el holandés Vonk y retomado por González, Araneda y Hernández (2005), denominado "nadar o hundirse", refleja la indiferencia y las con- diciones institucionales desfavorables por las cuales debe atravesar el docente en sus primeros años, en el proceso de inserción laboral.

Otra situación de tensión se produce cuando los profesores pretenden integrar las tecnologías de información y comunicación (TIC) a la enseñanza. Según Sánchez (2002), integrar las TIC al proceso de enseñanza implica empotrarlas en las metodologías y la didáctica con la intención de facilitar el aprendizaje en los alumnos. Esta tensión es generada por la falta de preparación docente en el uso de las TIC, por la resistencia de los alumnos a realizar trabajos educativos empleándolas y porque no todos ellos tienen acceso a las tecnologías. Además, la integración de las TIC no se hace efectiva en la práctica de estos docentes noveles, fundamentalmente, porque en estas prácticas opera un supuesto pedagógico didáctico que lo estaría obturando. La simultaneidad supone que la enseñanza tiene que ser llevada adelante del mismo modo para todos, todos aprenden las mismas cosas, al mismo tiempo y en el mismo lugar: el aula de clase. Esta es una idea fundacional de la escuela moderna con la cual es muy difícil romper (Terigi, 2010). En efecto, intentar integrar las TIC a la enseñanza implica cambiar esta matriz profundamente arraigada en nuestro sistema escolar, en razón de que las TIC amplían los límites del espacio y del tiempo de la escuela. La escuela ya no empieza ni termina en la puerta, y los horarios de clases van más allá de la hora de entrada y de salida. Una integración verdadera supone cambios profundos que conmuevan los supuestos que estructuran las formas de enseñar y de aprender

Una nueva tensión en la práctica escolar del profesor novel ocurre cuando evalúan los aprendizajes del alumnado. Las dificultades comunes prevalecientes están relacionadas con la evaluación diagnóstica y formativa, la corrección de exámenes escritos, emplear estrategias de evaluación alternativas y diseñar instrumentos. Asimismo, en sus respuestas se encuentran valoraciones predominantes de aspectos actitudinales que puedan exhibir los alumnos, como dedicación, esfuerzo, predisposición, 
asistencia, participación, etc. Estos hallazgos coinciden parcialmente con los informados por Contreras et al. (2012), quienes examinan las concepciones que subyacen a las respuestas brindadas por los profesores noveles al interrogante ¿qué, cómo y para qué evaluar? Sostienen estos que en sus planteamientos aparecen matices de una concepción tradicional sobre la evaluación, pues sus respuestas se orientan primordialmente a recuperar aspectos de medición y verificación de conocimientos acumulados, dejando de lado rasgos relativos al proceso y acompañamiento de las actividades de enseñanza y aprendizaje. En este análisis, dominan valoraciones de la dimensión declarativa del conocimiento antes que la actitudinal, que es la que se manifiesta en este artículo.

Finalmente, la dimensión relacional constituye otra fuente de tensión en la inserción laboral del profesor novel. En cuanto a las relaciones pedagógicas con los alumnos, las principales dificultades señaladas hacen referencia a mantener la disciplina en el aula y despertar el interés de los estudiantes. En el estudio llevado a cabo por Serra, Krichesky y Merodo (2009) con egresado de la Universidad Nacional de General Sarmiento en sus primeros desempeños laborales en secundarios del conurbano bonaerense, se arribó a análogos resultados; en este caso las dificultades fueron reunidas en la categoría manejo de grupos. El desinterés de los alumnos para emprender las actividades escolares y los episodios de indisciplina que interfieren en el desarrollo de la enseñanza del novel, se pueden caracterizar como un modo particular que adoptan numerosos jóvenes al transitar la escuela; desde el punto de vista sociológico, Kessler (2002) plantea la noción de escolaridad de baja intensidad para comprender este fenómeno. Básicamente, para afrontar tales dificultades, los profesores principiantes intentan mejorar su comunicación con los alumnos, articular acciones con los preceptores, realizar encuentros periódicos con los tutores, y también, una práctica bastante difundida, emplear la evaluación como mecanismo de control. La otra cara de la dimensión relacional son los vínculos profesionales que establece el profesor novel con sus pares, existe una tendencia general en estos profesores a buscar apoyo en sus colegas con mayor tiempo transcurrido en la docencia, se valoran los consejos y las orientaciones ya que brindan pistas de cómo resolver dificultades propias de la actividad docente. Sin embargo, también aparecen tensiones inherentes al vínculo mencionado, falta de compromiso institucional, individualismo, no hay sentido de pertenencia, egoísmo, falta disposición para el trabajo en equipo, ausentismo, etc. En este sentido, siguiendo los planteos de Terigi, se puede ubicar el origen de estas problemáticas en la organización del trabajo docente por horas de clases. Sostiene la autora que la colección de horas cátedra, que es la vinculación de la mayoría de los profesores, dificulta la concentración institucional, pues la unidad de designación no es el puesto en la escuela sino la asignatura (Terigi, 2008). El régimen laboral de los profesores aparece reportado en numerosos trabajos como uno de los principales problemas de las escuelas y como productor de otros problemas igualmente graves como los que fueron reseñados. Tal es la situación de los profesores noveles que trabajan sumando horas de clase en un recorrido por distintas instituciones educativas. Este hecho se agrava aún más en los profesores de Ciencias de la Educación dedicados a la docencia, ya que las asignaturas que pueden dictar están ubicadas en el tramo final de los planes de estudios del nivel medio, es decir, poseen pocas horas y, para completar la carga horaria, deben sumar escuelas; muchas veces en un mismo turno asisten a dos o tres escuelas ubicadas distantes espacialmente.

\section{Conclusiones}

Para concluir, esta investigación ofrece una primera aproximación al conocimiento de las experiencias por las que atraviesan profesores noveles en sus primeros años de ejercicio profesional. De acuerdo con investigaciones que se ocupan de la inserción laboral de este grupo profesional, se 
destaca la relevancia del acompañamiento y la generación de espacios de reflexión para resignificar y hacer consciente el efecto formativo de sus primeras experiencias laborales (Marcelo, 2007; Cornejo, 1999; Vaillant, 2005). Sin embargo, para las instituciones donde trabajan los profesores que formaron parte del estudio y la institución universitaria de la cual egresaron, aún no constituye una preocupación extendida ocuparse de los docentes que recién se inician.

Este trabajo muestra suficientes indicios para afirmar que el aprendizaje en la práctica generalmente está asociado a estrategias de supervivencia.
Esto se podría asociar a lo que se ha denominado shock de la realidad, que hace referencia al "colapso de los ideales misioneros elaborados durante la etapa de formación del profesorado con la cruda y dura realidad de la vida cotidiana en clase" (Veeman, 1988, p. 40).

En definitiva, esta conformidad estratégica de supervivencia profesional con el medio escolar, es la que distingue la socialización laboral de los egresados universitarios en sus primeros pasos en la docencia por el nivel medio, un pasaje que transcurre como un tiempo agitado de tensiones y aprendizajes intensos.

\section{Referencias}

Alonso Álvarez, E. (2001). Experiencia de formación permanente del profesorado de secundaria. Un itinerario de formación. En La formación del profesorado: proyectos de formación en centros educativos (pp. 129-138). Caracas: Laboratorio educativo.

Bleichmar, S. (1997) Acerca del malestar sobrante. Topia, 21. Recuperado el 23 de julio de 2014 de http://www. topia.com.ar/articulos/acerca-del-malestar-sobrante

Contreras Domingo, J. (1987) De estudiante a profesor. Socialización y aprendizaje en las prácticas de enseñanza. Educación, 282, 203-231.

Contreras, I., Rittershaussen, S., Solís, C., Núñez, C. y Vásquez, N. (2012). Cómo abordan profesores principiantes la evaluación de los aprendizajes de sus alumnos: un estudio de caso. III Congreso sobre profesorado principiante e inserción profesional a la docencia. Recuperado el 23 de julio de 2014 de http://prometeo.us.es/ congreso/comunicaciones/29/aula\%201/39.pdf

Cornejo Abarca, J. (1999). Profesores que se inician en la docencia: algunas reflexiones al respecto desde América Latina. Revista Iberoamericana de Educación, 19, 51-100. Recuperado el 23 de julio de 2014 de http://www. rieoei.org/oeivirt/rie19ao2.PDF Davini, M. (1995). La formación docente en cuestión: política y pedagogía.

Esteve, J., Franco, S. y Vera, J. (1995). Los profesores ante el cambio social. Barcelona: Anthropos.

Esteve, J. (1997). La formación inicial de los profesores de secundaria. Barcelona: Ariel.

Evans et al. (2010). Las prácticas de enseñanza en los egresados recientes del IFDC en Bariloche./l Congreso sobre profesorado principiante e inserción profesional a la docencia. Recuperado 25 de julio de 2014 de http:// cedoc.infd.edu.ar/noveles/principiantes/5/EXP_5_Evans_LAS_PRACTICAS_DE_ENSENANZA.pdf

González Brito, A., Araneda Garcés, N., Hernández González, J. (2005). Inducción profesional docente. Estudios peda-

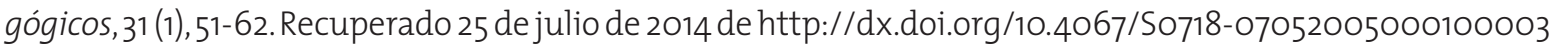


Huberman, M. (1989). La vie des enseingnants. Neuchâtel: Delachaux et Niestlé.

Imbernón, F. (1994). La formación y el desarrollo profesional del profesorado. Hacia una nueva cultura. Barcelona: Graó.

Kessler, G. (2002). La experiencia escolar fragmentada. Buenos Aires: IIPE-Unesco.

Lacey, C. (1977). The socialization of teachers. London: Methuen and co.

Marcelo García, C. y Estebaranaz García, A. (1998). Modelos de colaboración entre la Universidad y las escuelas en la formación inicial del profesorado. Educación, 317, 97-120.

Marcelo García, C. y Estebaranaz García, A. (1999). Formación de profesores para el cambio educativo. Barcelona: EUB.

Marcelo García, C. y Estebaranaz García, A. (2007). Empezar con buen pie: inserción a la enseñanza para profesores principiantes. Docencia, XII (33), 27-38. Recuperado 25 de julio de 2014 de http://www.oei.es/noticias/ spip.php?article2470

Rockwell, E. (1987). Desde la perspectiva del trabajo docente. Revista documentos DIE, 5, 1-32.

Sánchez, J. (2009). Integración curricular de las TIC: conceptos e ideas. Actas del VI Congreso Iberoamericano de Informática Educativa. Recuperado el o8 de agosto de 2014 de http://www.medellin.edu.co/sites/Educativo/ Docentes/primerclic/Evidencias\%20de\%20Aprendizaje1/Cohorte\%202\%202009/IntegracionCurriculardelasTICs.formato.pdf

Serra, J., Krichesky, G. y Merodo, A. (2009). Inserción laboral de docentes noveles del nivel medio en la argentina. Curriculum y formación del profesorado, 13 (1), 195-208.

Terigi, F. (2008). Los cambios en el formato de la escuela secundaria argentina: por qué son necesarios, por qué son tan difíciles. Propuesta Educativa, 17 (29), 63-71.

Terigi, F. (2010). Las cronologías de aprendizaje: un concepto para pensar las trayectorias escolares. Conferencia Jornada de apertura ciclo lectivo 2010. La Pampa. Recuperado el o8 de agosto de 2014 de http://www.lapampa.edu.ar:4040/rep/index.php/biblioteca-digital/conferencias/item/las-cronologias-de-aprendizaje-2

Vaillant, D. (2005). Formación de docentes en América Latina. Madrid: Octaedro.

Veeman, S. (1988). El proceso de llegar a ser: un análisis de la formación inicial. En Villa, A. (coord.). Perspectivas y problemas de la función docente (pp. 39-68). Madrid: Narcea.

Vera, J. (1988). La crisis de la función docente. Valencia: Promolibro.

Zeichner, K. M. (1993). Dialéctica de socialización del profesor. Educación, 52, 95-123.

Zoppi, A. M. (2001). La construcción social de la profesionalidad docente. Propuesta Educativa, 11 (24), 84-92. 
